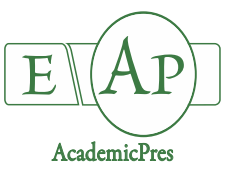

Chueakhunthod W et al. $(2020)$
Notulae Botanicae Horti Agrobotanici Cluj-Napoca 48(4):1845-1861
DOI:10.15835/48412114
Research Article

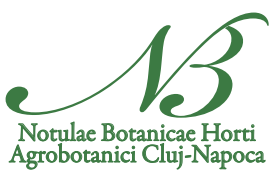

\title{
Genetic relationship of mungbean and blackgram genotypes based on agronomic and photosynthetic performance and SRAP markers
}

\author{
Witsarut CHUEAKHUNTHOD ${ }^{1}$, Wanploy JINAGOOL ${ }^{1}$, Kantima \\ MEECHAROEN ${ }^{1}$, Rungrapee KHWANMAN ${ }^{1}$, Pattaradol \\ PATTANARAM ${ }^{1}$, Natchaya JANTARAT ${ }^{1}$, Pratthana PALAPHON ${ }^{1}$, \\ Sumana NGAMPONGSAI ${ }^{2}$, Piyada A. TANTASAWAT ${ }^{1 *}$
}
${ }^{1}$ Suranaree University of Technology, School of Crop Production Technology, Institute of Agricultural Technology, 111 University Avenue, Muang District, Nakhon Ratchasima 30000,Thailand; witsarut.c@sut.ac.th;wanploy.jinagool@sut.ac.th;
khaikantima@gmail.com; rungrapee25@gmail.com; theparpit@gmail.com; thommaratmax@gmail.com; partt2539@gmail.com; piyada@sut.ac.th (*corresponding author)
${ }^{2}$ Field and Renewable Energy Crops Research Institute, 50 Phahonyothin Avenue, Chatuchak District, Bangkok 10900,
Thailand; sumana.56@hotmail.com

\begin{abstract}
Genetic identification is at the base of parental selection in varietal development programs. Agronomic and photosynthetic characters and sequence-related amplified polymorphism (SRAP) markers were analyzed for two legume species which included 23 mungbean (Vigna radiata (L.) Wilczek) and four blackgram (Vigna mungo (L.) Hepper) genotypes. The results revealed that the seeds/pod, plant height, pods/plant, pod length, days to flowering, and all photosynthetic characters studied had a significant correlation with the yield/plant. Using UPGMA analysis with phenotypic data, five clusters and two individuals were identified. Twenty-five SRAP primer combinations generated 562 amplified bands, of which 507 were polymorphic $(90.2 \%)$. The average numbers of scorable and polymorphic bands/primer pair were 22 and 20, respectively. Two major clusters coincided with two species having a $100 \%$ bootstrap value. Within the mungbean cluster, there were two subclusters containing 12 and 11 mungbean genotypes. Mantel's test demonstrated that the polymorphisms given by SRAPs were associated with agronomic and photosynthetic variability $(r=0.734, p<$ 0.01 ). These results allow promising mungbean genotypes to be identified through genetic diversity and field performance which can be utilized as potential parents towards future breeding programs. Moreover, the factors which contribute most to yield/plant can be simultaneously used as selection criteria for yield improvement.
\end{abstract}

Keywords: agronomic characters; cluster analysis; genetic diversity; photosynthesis; sequence-related amplified polymorphism; Vigna mungo(L.) Hepper; Vigna radiata (L.) Wilczek 


\section{Introduction}

In Thailand, the mungbean (Vigna radiata (L.) Wilczek) and blackgram (Vigna mungo (L.) Hepper) are considered to be strategic crops for local and national agribusiness. They contain significant amounts of enzymes, phytonutrients, and antioxidants within their dried seeds, forage or green/black pods, and fresh seeds, which are essential for maintaining better health (Somta and Srinives, 2007). The growing awareness of their benefits has led to an increased demand in recent years. However, production is not sufficient to keep up with demand. This is mainly because the existing varieties have a genetically low yield potential and are susceptible to biotic stresses (insect pests and diseases) and abiotic stresses (salinity, heat stress, waterlogging, and drought). Therefore, new varieties with an improved high yield potential and resistance to stresses are required as soon as possible. Finding suitable parents is the initial step in any breeding approach. Phenotyping is the usual way to allow breeders to select parents with the best characteristics. For these two Vigna species, only few studies have been conducted on photosynthetic performance diversity and relationships in combination with the agronomic characters (Hossain et al., 2009; Islam and Razzaque, 2010; Gao et al., 2015). With advances in molecular biology, DNA markers provide a feasible choice for assessing genetic information because they can overcome several limitations found by using phenotyping alone i.e., the magnitude of environmental factors on trait expression, the limited number of characters, and discrimination of genotypes with high phenotypic similarity (Smith and Smith, 1989). Recently, genome/transcriptome sequencing projects have evolved which influence a trend away from structural markers towards functional markers. Functional markers located near any genes are very useful for assessing functional diversity due to the polymorphisms generated from the exon and intron regions (Poczai et al., 2013). SRAP developed by Li and Quiros (2001) is a simple functional marker and has gained in popularity over other multilocus markers. Because of its simplicity, high reproducibility and because no prior genome sequence is needed, this method has become attractive for breeding programs. Aneja et al. (2013) and Alghamdi et al. (2017) found that all SRAP primer combinations exhibited 100\% polymorphism, thereby being useful in genetic assessment amongst mungbean accessions, as well as other Vigna spp. The objectives of this study were: i) to evaluate agronomic and photosynthetic performance of 23 mungbean and four blackgram genotypes and ii) to estimate their genetic diversity and relationships using SRAP markers.

\section{Materials and Methods}

\section{Plant materials}

A total of 23 mungbean and four blackgram genotypes were included in this study (Table 1). Among mungbean tested genotypes, five were Thai certified varieties that are popularly grown by Thai farmers; 'CN36', 'CN72', 'CN84-1', 'KPS1', and 'SUT1', and one was a Suranaree University of Technology (SUT) developed variety; 'SUT4' (Table 1). The first three varieties were developed by Chai Nat Field Crops Research Center, Thailand, while 'KPS1' and 'SUT1' were developed by Kasetsart University, Kamphaeng Saen Campus and Suranaree University of Technology, Thailand, respectively. Seventeen other mungbean genotypes were obtained from the World Vegetable Center in Taiwan. Four blackgram genotypes were also included, two of which were Thai certified varieties; 'CN2' and 'CN80' developed by the Chai Nat Field Crops Research Center, and the others were 'BR-1' and 'PAK40592' from the World Vegetable Center collection.

\section{Experimental site, design and crop establishment}

A field experiment for agronomic and photosynthetic characteristics was carried out at Suranaree University of Technology Farm, Nakhon Ratchasima, Thailand (latitude: 1452'39"N, longitude: 102 $00^{\prime} 15^{\prime \prime E}$, altitude: $227 \mathrm{~m}$ ) during March to June, 2020. The field trial was established in a randomized incomplete block design with four plots per genotype. Four replications were used when the plants of each 
genotype in each plot grew well all the way up to field characterization, while three replications were used if uneven growth was observed in one of the plots. In each replication, seeds were planted in two-meter-long rows with a spacing of 0.5 and $0.2 \mathrm{~m}$ between rows and between plants within rows, respectively, which was kept after thinning. Two seedlings were maintained per hill for each genotype. Agronomic practices were performed as described by Khajudparn (2009).

Table 1. Pedigree, origins, and special features of 23 mungbean and four blackgram genotypes used in this study

\begin{tabular}{|c|c|c|c|c|}
\hline Genotypes & Pedigree & Origins & Special features & Description \\
\hline \multicolumn{5}{|c|}{ Vigna radiata (L.) Wilczek } \\
\hline 'CN36’ & 'PAGASA1' × 'PHLV18' & Thailand & $\begin{array}{l}\text { Large seed, uniform maturity, } \\
\text { moderate resistance to PM and } \\
\text { CLS }\end{array}$ & \multirow{3}{*}{$\begin{array}{c}\text { Varieties developed at Chai Nat } \\
\text { Field Crops Research Center, } \\
\text { Thailand. }\end{array}$} \\
\hline 'CN72’ & $\begin{array}{c}\text { Selection from mutated } \\
\text { 'KPS2' [(BPI GLABROUS } \\
\# 3 \times(\text { ('CES44' × 'ML-3') }) \times \\
\text { 'CN36'] }\end{array}$ & Thailand & $\begin{array}{l}\text { High yield, suitability for all } \\
\text { conditions }\end{array}$ & \\
\hline ‘CN84-1’ & $\begin{array}{l}\text { Selection from mutated } \\
\text { 'CN36' }\end{array}$ & Thailand & $\begin{array}{l}\text { High yield, high percentage of } \\
\text { starch }\end{array}$ & \\
\hline 'KPS1' & 'PAGASA1' × 'EG-MG-16’ & Thailand & $\begin{array}{l}\text { Moderate resistance to PM and } \\
\text { CLS }\end{array}$ & $\begin{array}{c}\text { Kasetsart University, } \\
\text { Kamphaeng Saen Campus, } \\
\text { Thailand. }\end{array}$ \\
\hline 'SUT1' & 'UTHONG1’ × 'NP-29’ & Thailand & $\begin{array}{l}\text { High yield, high suitability for } \\
\text { harvest, resistance to PM and CLS }\end{array}$ & \multirow{2}{*}{$\begin{array}{c}\text { Varieties developed at Suranarec } \\
\text { University of Technology, } \\
\text { Thailand. }\end{array}$} \\
\hline 'SUT4' & $\begin{array}{c}\text { 'MV1' } \times(\text { ('MX 4-7 M0317' } \\
\text { ×'M0277') }\end{array}$ & Thailand & Resistance to PM and CLS & \\
\hline $\begin{array}{l}\text { 'V4718 } \\
\text { (PLM. 945)' }\end{array}$ & - & India & High resistance to PM and CLS & \multirow{17}{*}{$\begin{array}{c}\text { Lines/varieties obtained from } \\
\text { the World Vegetable Center, } \\
\text { Taiwan. }\end{array}$} \\
\hline $\begin{array}{c}\text { 'V4758 } \\
\text { (PLM. 994)' }\end{array}$ & - & India & High resistance to $\mathrm{PM}$ & \\
\hline $\begin{array}{c}\text { 'V4785 } \\
\text { (PLM. 1033)' }\end{array}$ & - & India & High resistance to $\mathrm{PM}$ & \\
\hline 'PUSA-105' & $\begin{array}{l}\text { ('TAINAN-1' × 'ML-6') } \times \\
\left({ }^{\prime} E G-M G-16 \text { × ×'ML-3') }\right.\end{array}$ & India & $\begin{array}{l}\text { Moderate resistance to PM, CLS, } \\
\text { and Mungbean Yellow Mosaic } \\
\text { Virus (MYMV) }\end{array}$ & \\
\hline 'ML-131' & 'ML-1' × 'ML-23' & India & Resistance to PM and MYMV & \\
\hline 'VAR A-G' & - & India & Resistance to PM & \\
\hline 'BARI MUNG 2' & 'M-7715' & Bangladesh & $\begin{array}{l}\text { Photo-insensitive, resistance to } \\
\text { MYMV and CLS }\end{array}$ & \\
\hline 'NM92' & 'LM641' × 'NM36' & Pakistan & Resistance to CLS and MYMV & \\
\hline 'NM94' & 'YEZIN MUNGBEAN 11' & Myanmar & Resistance to CLS and MYMV & \\
\hline 'EG-MD-6D’ & 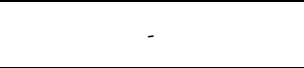 & Philippines & $\begin{array}{l}\text { High yield, general resistance to } \\
\text { diseases }\end{array}$ & \\
\hline 'CES55' & 'CES14' x 'MG50-10A' & Philippines & High yield & \\
\hline 'MG50-10A (Y)' & $\begin{array}{l}\text { 'GLOSSY GREEN S1’× } \\
\text { 'GLABROUS GREEN’ }\end{array}$ & Philippines & $\begin{array}{l}\text { High yield, photo-insensitive, } \\
\text { uniform maturity }\end{array}$ & \\
\hline $\begin{array}{l}\text { 'BPI GLABROUS } \\
\# 3 \text { ' }\end{array}$ & 'MG50-10A' × 'ILAG S-6A' & Philippines & Large seed, high yield & \\
\hline 'WALET' & 'EG-MG-4’ × 'ML-6’ & Indonesia & $\begin{array}{l}\text { High yield, resistance to PM and } \\
\text { CLS }\end{array}$ & \\
\hline 'GELATIK' & 'CES55' × 'ML-3' & Indonesia & Resistance to PM and CLS & \\
\hline 'KING' & 'EG-MG-7’ & Australia & $\begin{array}{c}\text { Large seed, high yield, resistance to } \\
\text { PM }\end{array}$ & \\
\hline 'TAINAN SEL\#5' & - & Taiwan & $\begin{array}{l}\text { High yield, resistance to PM and } \\
\text { CLS }\end{array}$ & \\
\hline \multicolumn{5}{|c|}{ Vigna mungo (L.) Hepper } \\
\hline 'CN2' & 'KAB4' × 'PLU1131' & Thailand & High yield & \multirow{2}{*}{$\begin{array}{c}\text { Varieties developed at Chai Nat } \\
\text { Field Crops Research Center, } \\
\text { Thailand. }\end{array}$} \\
\hline ‘CN80’ & 'PRAJEEN' × 'NBG' & Thailand & High yield, suitability for harvest & \\
\hline 'BR-1' & - & India & Resistance to bruchid & \multirow{2}{*}{$\begin{array}{c}\text { Lines/varieties obtained from } \\
\text { the World Vegetable Center, } \\
\text { Taiwan. }\end{array}$} \\
\hline 'PAK40592' & - & Pakistan & High yield & \\
\hline
\end{tabular}




\section{Agronomic and photosynthetic characterization}

Eleven quantitative agronomic traits were measured including the number of days to flowering, days to maturity, clusters/plant, branches/plant, plant height, pods/plant, pod length, pod width, 100 seed-weight, seeds/pod, and yield/plant. Measurement techniques followed those established by IBPGR Secretariat (1980) and Chai Nat Field Crops Research Center (2018). Ten randomly selected plants in the middle of each plot were used for measurement (Table 2). Four photosynthetic traits were measured with three plants in each plot, which were selected so as to be the same plants used for agronomic measurements on four consecutive cloudfree days. Disease responses were evaluated according to Chankaew et al. (2011) and Khajudparn et al. (2007) for Cercospora leaf spot (CLS) and powdery mildew (PM) in 2017 and 2018, respectively, when diseases were evenly spread across the fields. SPSS 16.0 software (SPSS Inc., Chicago, IL, USA) was used to perform an analysis of variance (ANOVA) followed by Duncan's post hoc statistical tools of agronomic and photosynthetic traits and disease responses. This software was also conducted to calculate correlation of agronomic and photosynthetic traits.

Table 2. Evaluation methods of agronomic and photosynthetic characters

\begin{tabular}{|c|c|c|}
\hline No. & Characters & Evaluation methods \\
\hline 1 & Days to flowering & $\begin{array}{l}\text { Number of days from sowing to } 50 \% \text { of plants in the plot with } \\
\text { first flower open. }\end{array}$ \\
\hline 2 & Days to maturity & $\begin{array}{l}\text { Number of days from sowing to } 50 \% \text { of plants in the plot with } \\
\text { first pod ripe. }\end{array}$ \\
\hline 3 & Clusters/plant & $\begin{array}{l}\text { Number of clusters having at least one fully grown pod at first } \\
\text { harvest including both main stem and branches. Average of ten } \\
\text { plants/plot. }\end{array}$ \\
\hline 4 & Branches/plant & $\begin{array}{l}\text { Number of pod-bearing branches with at least two nodes. Average } \\
\text { of ten plants/plot. }\end{array}$ \\
\hline 5 & Plant height $(\mathrm{cm})$ & $\begin{array}{l}\text { From soil level to the highest point after the first harvest. Average } \\
\text { of ten plants/plot. }\end{array}$ \\
\hline 6 & Pods/plant & Number of pods from two harvests. Average of ten plants/plot. \\
\hline 7 & Pod length $(\mathrm{cm})$ & $\begin{array}{c}\text { Maximum length of four to ten pods (in case of curved pods, the } \\
\text { longest straight line from the base to the tip of pod was } \\
\text { measured.). Average of ten plants/plot. }\end{array}$ \\
\hline 8 & Pod width $(\mathrm{mm})$ & Maximum width of four to ten pods. Average of ten plants/plot. \\
\hline 9 & 100-seed weight $(\mathrm{g})$ & $\begin{array}{l}\text { Weight of } 100 \text { randomly selected seeds. Average of ten plants/plot } \\
\text { (50 seeds from two plants were combined if necessary). }\end{array}$ \\
\hline 10 & Seeds/pod & $\begin{array}{l}\text { Number of seeds/pod of four to ten pods. Average of ten } \\
\text { plants/plot. }\end{array}$ \\
\hline 11 & Yield/plant $(\mathrm{g})$ & Total seed yield from two harvests. Average of ten plants/plot. \\
\hline 12 & Photosynthetic rate $(\mathrm{Pn})\left(\mu \mathrm{mol} \mathrm{m}{ }^{-2} \mathrm{~s}^{-1}\right)$ & \multirow{4}{*}{$\begin{array}{l}\text { Three uppers most fully-expanded terminal leaves of } 40 \text { days-old } \\
\text { plant (full bloom stage, R2 showing the greatest photosynthetic } \\
\text { potential in mungbean (Gao et al., 2015)) were measured by a } \\
\text { portable photosynthesis system (Model LCA-4, Hoddesdon, UK) } \\
\text { at 9:30 am to 2:30 pm. Average of three plants/plot. }\end{array}$} \\
\hline 13 & Transpiration rate $(\mathrm{Tr})\left(\mathrm{mmol} \mathrm{m} \mathrm{s}^{-1}\right)$ & \\
\hline 14 & Stomatal conductance $(\mathrm{Gs})\left(\mathrm{mol} \mathrm{m} \mathrm{s}^{-1}\right)$ & \\
\hline 15 & $\begin{array}{l}\text { Water use efficiency (WUE) } \\
\left(\mathrm{mmol} \mathrm{mol}^{-1}\right)\end{array}$ & \\
\hline 16 & Cercospora leaf spot response & Recorded from each plot in 2017. \\
\hline 17 & Powdery mildew response & Recorded from each plot in 2018. \\
\hline
\end{tabular}

\section{SRAP analysis}

Fresh young leaves from three individual plants of each genotype, reaching homozygosity whose uniformity had been previously checked, were taken and bulked. Genomic DNA was isolated according to the 
modified CTAB protocol of Lodhi et al. (1994). DNA concentration and purity were assessed by a ND-1000 spectrophotometer at $\mathrm{A}_{260}$ and $\mathrm{A}_{280}$ (NanoDrop Technologies, Inc., Wilmington, DE, USA). Five different forward and reverse primers were combined randomly to generate 25 SRAP primer combinations (Table 3). A polymerase chain reaction (PCR) was carried out in $20 \mu \mathrm{L}$ reaction volumes containing $150 \mathrm{ng}$ of DNA, $1 \mathrm{x}$ buffer (150 mM Tris-HCl, $\mathrm{pH} 8.75$ at $25^{\circ} \mathrm{C}, 500 \mathrm{mM} \mathrm{KCl}, 20 \mathrm{mM} \mathrm{MgCl}_{2}, 1 \%$ Triton X-100), $200 \mu \mathrm{M}$ of each deoxyribonucleotide triphosphate (dNTPs), $0.6 \mu \mathrm{M}$ of each forward and reverse primer, and $1 \mathrm{U}$ of Taq DNA polymerase (Vivantis, Selangor Darul Ehsan, Malaysia). The PCR conditions according to Aneja et al. (2013) were followed. T100 ${ }^{\mathrm{TM}}$ Thermal Cycler (Bio-rad Laboratory, Inc., California, USA) was used for DNA amplification. The PCR products were separated on $6 \%$ denaturing polyacrylamide gel at $200 \mathrm{~V}$ using vertical electrophoresis for $70 \mathrm{~min}$. The gel was stained using silver nitrate (Sambrook and Russell, 2001). The molecular weights of the DNA bands were estimated by comparison with a 100 bp DNA Ladder as a marker (Invitrogen, California, USA).

Table 3. List of sequence-related amplified polymorphism (SRAP) primer combinations used in the study

\begin{tabular}{|c|c|c|c|c|}
\hline SRAP primer combinations & $\begin{array}{c}\text { Total } \\
\text { fragments }\end{array}$ & Polymorphic fragments (\%) & Band size range (bp) & PIC \\
\hline me1(5'-BATA-3')-em1(5'-DAAT-3') & 21 & 81 & $200-1,000$ & 0.228 \\
\hline me1(5'-BATA-3')-em2(5'-DTGC-3') & 38 & 100 & $200-1,100$ & 0.296 \\
\hline me1(5'-BATA-3')-em3(5'-DGAC-3') & 23 & 91 & $200-700$ & 0.232 \\
\hline mel(5'-BATA-3')-em4(5'-DTGA-3') & 23 & 83 & $200-1,200$ & 0.269 \\
\hline mel(5'-BATA-3')-em5(5'-DAAC-3') & 20 & 85 & $200-700$ & 0.225 \\
\hline me2(5'-BAGC-3')-em1(5'-DAAT-3') & 27 & 96 & $200-800$ & 0.252 \\
\hline me2(5'-BAGC-3')-em2(5'-DTGC-3') & 21 & 81 & $200-1,400$ & 0.303 \\
\hline me2(5'-BAGC-3')-em3(5'-DGAC-3') & 14 & 50 & $200-700$ & 0.170 \\
\hline me2(5'-BAGC-3')-em4(5'-DTGA-3') & 15 & 80 & $200-700$ & 0.221 \\
\hline me2(5'-BAGC-3')-em5(5'-DAAC-3') & 23 & 87 & $200-1,400$ & 0.247 \\
\hline me3(5'-BAAT-3')-em1(5'-DAAT-3') & 21 & 100 & $200-900$ & 0.277 \\
\hline me3(5'-BAAT-3')-em2(5'-DTGC-3') & 20 & 100 & $200-700$ & 0.230 \\
\hline me3(5'-BAAT-3')-em3(5'-DGAC-3') & 20 & 90 & $200-1,000$ & 0.304 \\
\hline me3(5'-BAAT-3')-em4(5'-DTGA-3') & 22 & 100 & $200-1,400$ & 0.236 \\
\hline me3(5'-BAAT-3')-em5(5'-DAAC-3') & 25 & 84 & $200-900$ & 0.253 \\
\hline me4(5'-BACC-3')-em 1(5'-DAAT-3') & 29 & 93 & $200-900$ & 0.314 \\
\hline me4(5'-BACC-3')-em2(5'-DTGC-3') & 28 & 93 & $200-1,400$ & 0.260 \\
\hline me4(5'-BACC-3')-em3(5'-DGAC-3') & 19 & 100 & $200-900$ & 0.289 \\
\hline me4(5'-BACC-3')-em4(5'-DTGA-3') & 21 & 100 & $200-1,000$ & 0.280 \\
\hline me4(5'-BACC-3')-em5(5'-DAAC-3') & 16 & 100 & $200-800$ & 0.267 \\
\hline me5(5'-BAAG-3')-em1(5'-DAAT-3') & 15 & 87 & $200-800$ & 0.189 \\
\hline me5(5'-BAAG-3')-em2(5'-DTGC-3') & 27 & 89 & $200-900$ & 0.244 \\
\hline me5(5'-BAAG-3')-em3(5'-DGAC-3') & 23 & 87 & $200-1,300$ & 0.283 \\
\hline me5(5'-BAAG-3')-em4(5'-DTGA-3') & 17 & 71 & $200-1,000$ & 0.274 \\
\hline me5(5'-BAAG-3')-em5(5'-DAAC-3') & 34 & 97 & $200-900$ & 0.324 \\
\hline Total & 562 & & & \\
\hline Average & 22 & 89 & & 0.259 \\
\hline
\end{tabular}

Notes: $\mathrm{B}$ = TGAGTCCAAACCGG, D = GACTGCGTACGAATT. PIC = Polymorphic information content

\section{Data analyses}

The SRAP amplified bands were coded as "0" and " 1 " for their absence or presence, respectively, similar to other dominant markers. PIC was calculated according to PIC $=1-\Sigma \mathrm{Pi}^{2}$, where $\mathrm{i}$ is the total number of alleles detected for the SRAP marker, and Pi is the frequency of the $\mathrm{i}^{\text {th }}$ allele in the genotypes studied. The unweighted pair group method average (UPGMA) dendrogram of agronomic and photosynthetic characters and polymorphic SRAP loci was constructed based on Euclidean distance to measure the genetic dissimilarity coefficients in a pair-wise comparison across all genotypes using PAST software (version 4.03) with a bootstrap frequency of 1000 . The goodness of fit for the genotypes to a specific cluster in the UPGMA algorithm was 
executed by the normalized Mantel statistic $Z$ test (Mantel, 1967). Correlation between two cophenetic metrics from agronomic and photosynthetic characters and SRAP markers was analyzed based on the Mantel matrix correspondence test through XLSTAT software (version 2015) (Addinsoft, Inc., Paris, France) (Mantel, 1967). A correlation value $(\mathrm{r})$ appearing greater than 0.5 indicates that there is a statistical significance at 0.01 probability level, if over 15 taxonomic units are observed (Lapointe and Legendre, 1992). PAST software (version 4.03) was used again to construct principal coordinate analysis (PCoA) to represent the clustering pattern of genotypes in the first two principal coordinates, which accounted for the highest variation.

\section{Results and Discussion}

\section{Agronomic and photosynthetic character analysis}

The mungbean and blackgram genotypes displayed significant differences for all of their agronomic characters and for the two disease responses $(p<0.01)$ but not for their photosynthetic characters $(p>0.05)$ (Tables 4 and 5). Mungbean genotypes with desirable characters were observed as follows: early flowering and maturity (i.e. 'NM94', 'V4718', 'PUSA-105', and 'ML-131'); resistance to diseases ('V4718', 'V4758', and 'V4785') and high yielding potential with high pods/plant ('ML-131' and 'BARI MUNG2'), high pod length ('EG-MD-6D', 'CES55', 'TAINAN SEL\#5', 'BPI GLABROUS \#3', 'KING', and 'MG50-10A (Y)'), high pod width ('CN36', 'CN72', 'KING', 'MG50-10A (Y)', 'BPI GLABROUS \#3', 'CN84-1', 'TAINAN SEL\#5', and 'EG-MD-6D'), high 100-seed weight ('MG50-10A (Y)', 'BPI GLABROUS \#3', 'KING', and 'EG-MD-6D'), and high seeds/pod ('VAR A-G', 'V4758', or 'BARI MUNG2').

Crop yield implicates a variety of other contributing component characters. An analysis of the correlation between yield and its independent variables can potentially identify their relative significance to improve higher yield. In this analysis, yield/plant corresponded to several agronomic characters: seeds/pod ( $\mathrm{r}$ $\left.=0.612^{* \prime}\right)$, plant height $\left(\mathrm{r}=0.569^{\circ *}\right)$, pods/plant $\left(\mathrm{r}=0.435^{*}\right)$, pod length $\left(\mathrm{r}=0.430^{* \prime}\right)$, and days to flowering $(\mathrm{r}$ $\left.=0.200^{\circ}\right)$, as well as all photosynthetic characters studied including Pn $\left(r=0.463^{*}\right)$, Gs $\left(r=0.406^{\prime \prime}\right)$, WUE $(r$ $\left.=0.235^{\circ}\right)$, and $\operatorname{Tr}\left(\mathrm{r}=0.210^{\circ}\right)$ (Table 6), indicating that increasing these characters can possibly improve yield. However, days to maturity, clusters/plant, branches/plant, pod width, and 100-seed weight showed no correlation with yield. Most previous reports of yield and agronomic character relationships in mungbean also found that seeds/pod and pods/plant exhibited strong positive correlation with yield, while the associations with other characters, i.e. days to flowering, plant height, or pod length were not consistent among different works (Khattak et al., 1995; Khajudparn and Tantasawat, 2011; Mondal et al., 2011; Das and Barua, 2015; Hemavathy et al., 2015; Garg et al., 2017; Kumar et al., 2018; Manivelan et al., 2019; Tahir et al., 2020). This may be largely due to the differences in the plant materials, range of the characters, and evaluation time or environmental conditions studied in each work. For yield and photosynthetic character relationships, Srinivasan et al. (1985) demonstrated that photosynthetic rate was significantly associated with seed yield of mungbean at early pod development but not at the vegetative stage. However, Islam and Razzaque (2010) found that Pn, Gs, Tr, and WUE were not correlated with seed yield of mungbeans. It should be noticed that the photosynthetic rate and its related factors are not always correlated with crop yield (Curtis et al., 1969; Rhodes, 1972; Long et al., 2006). These inconsistent attributes may result from the high vulnerability of the plant's photosynthetic process to changes in environmental factors over a short period of time under evaluation or because of changes in photosynthesis and yield relatedness with growth stages (Islam et al., 1994). In addition to the relationships between yield/plant, the interrelationships between other characters were also evaluated. For example, days to flowering was significantly positively related to days to maturity, plant height, pod length, seeds/pod, and Gs, suggesting that selection for early genotypes may reduce plant height, pod length, seeds/pod and Gs. 
Table 4. Agronomic characters of 23 mungbean and four blackgram genotypes

\begin{tabular}{|c|c|c|c|c|c|c|c|c|c|c|c|c|c|}
\hline Genotypes & 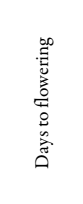 & 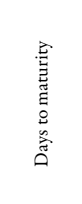 & 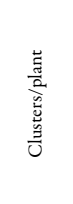 & 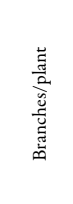 & 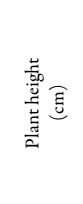 & $\begin{array}{l}\frac{n}{0} \\
\frac{a}{2} \\
\frac{5}{0} \\
0\end{array}$ & 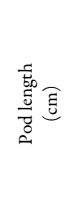 & 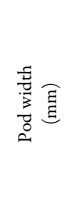 & 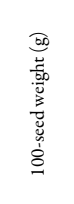 & 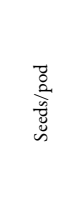 & 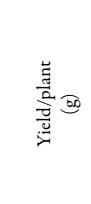 & 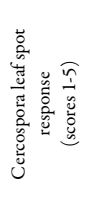 & 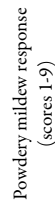 \\
\hline 'CN36' & $40.00 \mathrm{a}-\mathrm{e}$ & $\begin{array}{c}55.25 \mathrm{bc} \\
\mathrm{d}\end{array}$ & $6.43 \mathrm{f}-\mathrm{i}$ & $0.27 \mathrm{k}$ & $71.08 \mathrm{abc}$ & $18.97 \mathrm{~g}-\mathrm{k}$ & $9.56 \mathrm{~b}-\mathrm{e}$ & $6.68 \mathrm{a}$ & $7.05 \mathrm{~d}$ & $10.40 \mathrm{~b}-\mathrm{e}$ & $10.77 \mathrm{abc}$ & $5.00 \mathrm{a}$ & $\begin{array}{c}7.00 \\
a-d\end{array}$ \\
\hline 'CN72' & $39.25 a-f$ & $54.75 \mathrm{cde}$ & $5.95 \mathrm{ghi}$ & $0.25 \mathrm{k}$ & $59.42 \mathrm{a}-\mathrm{f}$ & $15.44 \mathrm{ijk}$ & $9.29 \mathrm{de}$ & $6.64 \mathrm{ab}$ & $7.24 \mathrm{~cd}$ & $9.82 \mathrm{~d}-\mathrm{g}$ & $8.12 \mathrm{cde}$ & $5.00 \mathrm{a}$ & $\begin{array}{c}7.00 \\
\mathrm{a}-\mathrm{d}\end{array}$ \\
\hline 'CN84-1' & $40.75 \mathrm{ab}$ & $55.50 \mathrm{bc}$ & $6.82 \mathrm{e}-\mathrm{i}$ & $0.79 \mathrm{~h}-\mathrm{k}$ & $70.43 \mathrm{abc}$ & $18.03 \mathrm{~h}-\mathrm{k}$ & $9.47 \mathrm{cde}$ & $6.54 a-d$ & $7.06 \mathrm{~d}$ & $10.08 \mathrm{c}-\mathrm{f}$ & $9.58 \mathrm{a}-\mathrm{e}$ & $5.00 \mathrm{a}$ & $\begin{array}{l}6.33 \\
\text { cde }\end{array}$ \\
\hline 'KPS1' & $39.25 a-f$ & $54.50 \mathrm{cde}$ & $7.32 \mathrm{e}-\mathrm{i}$ & $1.29 \mathrm{fgh}$ & $67.14 a-d$ & $21.00 f-k$ & $9.13 \mathrm{e}$ & $6.31 \mathrm{~b}-\mathrm{f}$ & $6.37 \mathrm{f}$ & $9.71 \mathrm{~d}-\mathrm{g}$ & $9.12 \mathrm{~b}-\mathrm{e}$ & $4.67 a$ & $\begin{array}{l}6.67 \\
b-e\end{array}$ \\
\hline 'SUT1' & $40.50 \mathrm{abc}$ & $\begin{array}{c}55.25 \\
\text { bcd }\end{array}$ & $7.68 \mathrm{e}-\mathrm{i}$ & $1.23 \mathrm{fgh}$ & $72.82 \mathrm{ab}$ & $25.70 \mathrm{c}-\mathrm{i}$ & $9.51 \mathrm{~b}-\mathrm{e}$ & $6.20 \mathrm{~d}-\mathrm{g}$ & $6.88 \mathrm{de}$ & $9.54 \mathrm{~d}-\mathrm{g}$ & $12.23 \mathrm{ab}$ & $4.00 \mathrm{~b}$ & $\begin{array}{c}5.67 \\
\mathrm{de}\end{array}$ \\
\hline 'SUT4' & 39.33a-f & $54.67 \mathrm{cde}$ & $5.75 \mathrm{hi}$ & $0.48 \mathrm{ijk}$ & $50.21 \mathrm{~d}-\mathrm{i}$ & $18.06 \mathrm{~h}-\mathrm{k}$ & $9.18 \mathrm{de}$ & $6.23 \mathrm{c}-\mathrm{g}$ & $6.51 \mathrm{lef}$ & $9.58 \mathrm{~d}-\mathrm{g}$ & $8.51 \mathrm{cde}$ & $4.67 \mathrm{a}$ & $\begin{array}{c}5.67 \\
\text { de }\end{array}$ \\
\hline 'V4718' & $34.25 \mathrm{hi}$ & $48.00 \mathrm{~g}$ & $8.70 \mathrm{~d}-\mathrm{h}$ & 1.01ghi & $55.46 \mathrm{c}-\mathrm{h}$ & $32.29 \mathrm{~cd}$ & $6.15 \mathrm{i}$ & $4.73 \mathrm{kl}$ & 3.061 & $10.58 \mathrm{~b}-\mathrm{e}$ & $7.67 \mathrm{c}-\mathrm{f}$ & $3.33 c$ & $\begin{array}{c}4.00 \\
\mathrm{~g}\end{array}$ \\
\hline 'V4758’ & $41.00 \mathrm{ab}$ & $58.00 \mathrm{a}$ & $8.87 \mathrm{~d}-\mathrm{g}$ & $2.26 \mathrm{~cd}$ & $56.84 \mathrm{~b}-\mathrm{g}$ & $31.46 \mathrm{cde}$ & $7.16 \mathrm{~h}$ & $4.74 \mathrm{kl}$ & 3.231 & $11.36 \mathrm{ab}$ & $9.24 \mathrm{~b}-\mathrm{e}$ & $2.33 \mathrm{~d}$ & $\begin{array}{l}4.33 \\
\mathrm{fg}\end{array}$ \\
\hline 'V4785' & $41.75 \mathrm{a}$ & $57.00 \mathrm{ab}$ & $9.01 \mathrm{def}$ & $2.28 \mathrm{~cd}$ & $54.17 \mathrm{c}-\mathrm{i}$ & $27.73 \mathrm{c}-\mathrm{h}$ & $7.47 \mathrm{gh}$ & $4.97 \mathrm{i}-1$ & $3.88 \mathrm{j}$ & $10.41 \mathrm{~b}-\mathrm{e}$ & $8.69 \mathrm{cde}$ & $2.33 \mathrm{~d}$ & $\begin{array}{c}2.33 \\
\mathrm{~h}\end{array}$ \\
\hline 'PUSA-105' & $35.75 \mathrm{gh}$ & $48.50 \mathrm{fg}$ & $9.55 \mathrm{cde}$ & $1.57 \mathrm{efg}$ & $44.61 \mathrm{e}-\mathrm{i}$ & $33.83 \mathrm{c}$ & $7.66 \mathrm{gh}$ & $4.86 \mathrm{jkl}$ & $3.82 \mathrm{jk}$ & $10.44 \mathrm{~b}-\mathrm{e}$ & $9.33 \mathrm{~b}-\mathrm{e}$ & $4.67 \mathrm{a}$ & $\begin{array}{c}5.67 \\
\mathrm{de}\end{array}$ \\
\hline 'ML-131' & $36.50 \mathrm{fgh}$ & $50.25 \mathrm{f}$ & $15.27 \mathrm{a}$ & $2.68 \mathrm{c}$ & $51.87 \mathrm{~d}-\mathrm{i}$ & $56.50 \mathrm{a}$ & $6.41 \mathrm{i}$ & $4.81 \mathrm{jkl}$ & $3.33 \mathrm{kl}$ & $8.82 \mathrm{~g}$ & $10.05 a-e$ & $5.00 \mathrm{a}$ & $\begin{array}{c}7.00 \\
a-d \\
\end{array}$ \\
\hline 'VAR A-G' & $40.50 \mathrm{abc}$ & $54.25 \mathrm{cde}$ & $14.96 \mathrm{a}$ & $2.13 \mathrm{cde}$ & $75.24 a$ & $35.57 \mathrm{c}$ & $7.83 \mathrm{~g}$ & 4.661 & $3.35 \mathrm{kl}$ & $11.80 \mathrm{a}$ & $10.69 \mathrm{abc}$ & $4.00 \mathrm{~b}$ & $\begin{array}{c}8.33 \\
\mathrm{a} \\
\end{array}$ \\
\hline 'BARI MUNG 2' & $37.33 \mathrm{~d}-\mathrm{g}$ & $53.00 \mathrm{de}$ & $13.90 \mathrm{ab}$ & $2.37 \mathrm{~cd}$ & $57.90 \mathrm{~b}-\mathrm{g}$ & $47.63 \mathrm{~b}$ & $7.35 \mathrm{gh}$ & $4.78 \mathrm{kl}$ & $3.49 \mathrm{jkl}$ & $11.18 \mathrm{abc}$ & $12.68 \mathrm{a}$ & $5.00 \mathrm{a}$ & $\begin{array}{c}8.00 \\
\mathrm{ab}\end{array}$ \\
\hline 'NM92' & $38.67 \mathrm{~b}-\mathrm{g}$ & $53.33 \mathrm{cde}$ & $7.06 \mathrm{e}-\mathrm{i}$ & $0.97 \mathrm{ghi}$ & $57.87 \mathrm{~b}-\mathrm{g}$ & $23.91 \mathrm{~d}-\mathrm{j}$ & $9.60 \mathrm{~b}-\mathrm{e}$ & $5.93 \mathrm{gh}$ & $6.17 \mathrm{f}$ & $9.68 \mathrm{~d}-\mathrm{g}$ & $10.69 \mathrm{abc}$ & $4.67 \mathrm{a}$ & $\begin{array}{l}6.00 \\
\text { cde }\end{array}$ \\
\hline 'NM94' & $31.50 \mathrm{j}$ & $46.50 \mathrm{~g}$ & $6.75 \mathrm{e}-\mathrm{i}$ & $0.45 \mathrm{ijk}$ & $43.31 \mathrm{f}-\mathrm{i}$ & $23.20 \mathrm{~d}-\mathrm{j}$ & $9.49 \mathrm{~b}-\mathrm{e}$ & $5.79 \mathrm{~h}$ & $5.68 \mathrm{gh}$ & $10.19 \mathrm{~b}-\mathrm{f}$ & $9.34 \mathrm{~b}-\mathrm{e}$ & $5.00 \mathrm{a}$ & $\begin{array}{l}7.33 \\
\mathrm{abc} \\
\end{array}$ \\
\hline 'EG-MD-6D' & $38.50 \mathrm{~b}-\mathrm{g}$ & $54.00 \mathrm{cde}$ & $5.83 \mathrm{ghi}$ & $0.52 \mathrm{ijk}$ & $67.55 \mathrm{a}-\mathrm{d}$ & $15.52 \mathrm{ijk}$ & $10.28 \mathrm{a}$ & $6.44 a-e$ & $7.71 b c$ & $10.12 \mathrm{c}-\mathrm{f}$ & $9.19 \mathrm{~b}-\mathrm{e}$ & $4.67 \mathrm{a}$ & $\begin{array}{l}7.33 \\
\mathrm{abc} \\
\end{array}$ \\
\hline 'CES55' & $40.00 \mathrm{a}-\mathrm{e}$ & $\begin{array}{c}55.25 \\
\text { bcd }\end{array}$ & $7.47 \mathrm{e}-\mathrm{i}$ & $1.23 \mathrm{fgh}$ & 61.30a-e & $19.54 \mathrm{~g}-\mathrm{k}$ & $10.25 \mathrm{a}$ & $6.08 \mathrm{fgh}$ & $7.08 \mathrm{~d}$ & $\begin{array}{c}10.69 \\
\text { bcd }\end{array}$ & $10.45 \mathrm{a}-\mathrm{d}$ & $5.00 \mathrm{a}$ & $\begin{array}{l}6.00 \\
\text { cde }\end{array}$ \\
\hline 'MG50-10A (Y)' & $38.00 \mathrm{~b}-\mathrm{g}$ & $53.33 \mathrm{cde}$ & $5.20 \mathrm{i}$ & $0.20 \mathrm{k}$ & $46.12 \mathrm{e}-\mathrm{i}$ & $12.09 \mathrm{k}$ & $9.77 \mathrm{a}-\mathrm{d}$ & $6.55 \mathrm{abc}$ & $8.27 \mathrm{a}$ & $9.16 \mathrm{fg}$ & $7.25 \mathrm{def}$ & $5.00 \mathrm{a}$ & $\begin{array}{l}6.67 \\
\text { b-e }\end{array}$ \\
\hline $\begin{array}{c}\text { 'BPI } \\
\text { GLABROUS \#3' }\end{array}$ & $37.67 \mathrm{c}-\mathrm{g}$ & $53.67 \mathrm{cde}$ & $5.72 \mathrm{hi}$ & $0.35 \mathrm{jk}$ & $55.72 \mathrm{~b}-\mathrm{h}$ & $15.49 \mathrm{ijk}$ & $9.98 \mathrm{abc}$ & $6.55 \mathrm{abc}$ & $7.79 \mathrm{~b}$ & $9.78 \mathrm{~d}-\mathrm{g}$ & $8.68 \mathrm{cde}$ & $5.00 \mathrm{a}$ & $\begin{array}{c}7.00 \\
a-d\end{array}$ \\
\hline 'WALET' & 39.75a-e & $54.50 \mathrm{cde}$ & $6.18 \mathrm{f}-\mathrm{i}$ & $0.88 \mathrm{hij}$ & $59.34 \mathrm{a}-\mathrm{f}$ & $23.00 \mathrm{~d}-\mathrm{j}$ & $9.01 \mathrm{e}$ & $6.13 \mathrm{e}-\mathrm{h}$ & $6.55 \mathrm{ef}$ & $9.48 \mathrm{~d}-\mathrm{g}$ & $10.56 \mathrm{a}-\mathrm{d}$ & $5.00 \mathrm{a}$ & $\begin{array}{l}6.00 \\
\text { cde }\end{array}$ \\
\hline 'GELATIK' & $38.25 \mathrm{~b}-\mathrm{g}$ & $54.25 \mathrm{cde}$ & $9.06 \mathrm{def}$ & $1.79 \mathrm{def}$ & $54.52 \mathrm{c}-\mathrm{i}$ & $28.77 \mathrm{c}-\mathrm{g}$ & $8.50 \mathrm{f}$ & $5.84 \mathrm{~h}$ & $5.66 \mathrm{gh}$ & $9.41 \mathrm{efg}$ & $10.72 \mathrm{abc}$ & $4.67 \mathrm{a}$ & $\begin{array}{c}5.33 \\
\text { ef }\end{array}$ \\
\hline 'KING' & $40.25 \mathrm{a}-\mathrm{d}$ & $54.50 \mathrm{cde}$ & $5.08 \mathrm{i}$ & $0.36 \mathrm{jk}$ & $59.32 \mathrm{a}-\mathrm{f}$ & $14.36 \mathrm{jk}$ & $9.98 \mathrm{abc}$ & $6.62 \mathrm{ab}$ & $7.73 \mathrm{bc}$ & $9.39 \mathrm{efg}$ & $8.01 \mathrm{cde}$ & $5.00 \mathrm{a}$ & $\begin{array}{l}6.67 \\
\text { b-e }\end{array}$ \\
\hline $\begin{array}{l}\text { 'TAINAN } \\
\text { SEL\#5' }\end{array}$ & $37.00 \mathrm{efg}$ & $54.33 \mathrm{cde}$ & $7.80 \mathrm{e}-\mathrm{i}$ & $1.23 \mathrm{fgh}$ & $50.58 \mathrm{~d}-\mathrm{i}$ & $20.13 \mathrm{f}-\mathrm{k}$ & $10.07 \mathrm{ab}$ & $6.53 \mathrm{a}-\mathrm{d}$ & $6.14 \mathrm{fg}$ & $10.53 \mathrm{~b}-\mathrm{e}$ & $10.18 \mathrm{a}-\mathrm{d}$ & $3.33 \mathrm{c}$ & $\begin{array}{l}6.00 \\
\text { cde }\end{array}$ \\
\hline 'CN2' & $33.00 \mathrm{ij}$ & $54.33 \mathrm{cde}$ & $12.01 \mathrm{bc}$ & $2.30 \mathrm{~cd}$ & $42.58 \mathrm{f}-\mathrm{i}$ & $27.03 \mathrm{c}-\mathrm{h}$ & $4.80 \mathrm{jk}$ & $5.22 \mathrm{i}$ & $5.56 \mathrm{~h}$ & $5.51 \mathrm{i}$ & $6.78 \mathrm{efg}$ & $2.00 \mathrm{~d}$ & $\begin{array}{c}8.00 \\
a b\end{array}$ \\
\hline 'CN80' & $37.67 \mathrm{c}-\mathrm{g}$ & $57.00 \mathrm{ab}$ & $14.97 \mathrm{a}$ & $4.41 \mathrm{a}$ & 41.07ghi & $29.81 \mathrm{c}-\mathrm{f}$ & $4.94 \mathrm{j}$ & $5.15 \mathrm{ij}$ & $5.18 \mathrm{~h}$ & $6.60 \mathrm{~h}$ & $8.45 \mathrm{cde}$ & $2.00 \mathrm{~d}$ & $\begin{array}{l}6.00 \\
\text { cde }\end{array}$ \\
\hline 'BR-1' & $40.67 \mathrm{abc}$ & $59.00 \mathrm{a}$ & $10.94 \mathrm{~cd}$ & $3.36 \mathrm{~b}$ & $38.89 \mathrm{hi}$ & $23.15 \mathrm{~d}-\mathrm{j}$ & $4.37 \mathrm{k}$ & $5.07 \mathrm{ijk}$ & $4.59 \mathrm{i}$ & $5.43 \mathrm{i}$ & $4.67 \mathrm{fg}$ & $1.00 \mathrm{a}$ & $\begin{array}{l}6.33 \\
\text { cde }\end{array}$ \\
\hline 'PAK40592' & $33.00 \mathrm{ij}$ & $52.67 \mathrm{e}$ & $10.93 \mathrm{~cd}$ & $2.12 \mathrm{cde}$ & $37.81 \mathrm{i}$ & $21.49 \mathrm{e}-\mathrm{k}$ & $4.43 \mathrm{jk}$ & $5.28 \mathrm{i}$ & $5.46 \mathrm{~h}$ & $4.81 \mathrm{i}$ & $4.23 \mathrm{~g}$ & $2.00 \mathrm{~d}$ & $\begin{array}{c}8.33 \\
\mathrm{a}\end{array}$ \\
\hline
\end{tabular}

Notes: CLS and PM were evaluated in 2017 and 2018, respectively. CLS infection was scored at 65 days after sowing (DAS) on a scale of $1-5$, where $1=$ no disease symptom, $2=1-25 \%$ of total leaf area infected, $3=26-50 \%$ of total leaf area infected, $4=51-75 \%$ of total leaf area infected, and 5=76-100\% of total leaf area infected. PM infection was scored at 65 DAS on a scale of 1-9, where $1=$ no disease symptom, $2=2-3$ lesions on the lower part of leaves, $3=2-3$ lesions on the lower part of leaves, where spore formation can be observed, $4=$ full spore formation on the lower part of leaves, and a few lesions can be observed on the middle part of leaves, $5=$ like number 4 , but chlorosis leaves and much of spore formation can be observed, $6=$ like number 5 , but full spore formation can be observed, $7=$ spore formation on all parts of leaves, and $25 \%$ dry leaves can be observed, $8=$ like number 7 , but $25-50 \%$ dry leaves can be observed, and $9=$ like number 7 , but over $50 \%$ dry leaves can be observed. Means within the same column not showing the same letters are significantly different $(\mathrm{p} \leq 0.05)$ based on DMRT. 
Table 5. Photosynthetic characters of 23 mungbean and four blackgram genotypes

\begin{tabular}{|c|c|c|c|c|}
\hline Genotypes & $\operatorname{Pn}\left(\mu \mathrm{molm}^{-2} \mathrm{~s}^{-1}\right)$ & $\operatorname{Tr}\left(\mathrm{mmol} \mathrm{m} \mathrm{m}^{-2} \mathrm{~s}^{-1}\right)$ & $\mathrm{Gs}\left(\mathrm{mol} \mathrm{m}^{-2} \mathrm{~s}^{-1}\right)$ & WUE $\left(\mathrm{mmol} \mathrm{mol}^{-1}\right)$ \\
\hline 'CN36’ & 13.46 & 1.13 & 0.92 & 13.24 \\
\hline 'CN72’ & 13.65 & 1.20 & 0.83 & 12.24 \\
\hline 'CN84-1' & 15.38 & 1.49 & 0.86 & 10.70 \\
\hline 'KPS1' & 13.97 & 1.60 & 0.77 & 9.80 \\
\hline 'SUT1' & 17.65 & 1.55 & 0.84 & 12.06 \\
\hline 'SUT4' & 13.83 & 1.39 & 0.58 & 10.56 \\
\hline 'V4718' & 13.05 & 1.23 & 0.63 & 10.86 \\
\hline 'V4758' & 15.63 & 1.21 & 0.87 & 14.86 \\
\hline 'V4785' & 12.90 & 1.56 & 0.78 & 8.25 \\
\hline 'PUSA-105' & 13.94 & 1.69 & 0.63 & 10.46 \\
\hline 'ML-131' & 15.25 & 1.35 & 0.79 & 12.14 \\
\hline 'VAR A-G' & 14.09 & 1.35 & 0.63 & 10.66 \\
\hline 'BARI MUNG 2' & 16.70 & 1.73 & 0.78 & 9.54 \\
\hline 'NM92' & 15.92 & 1.21 & 0.79 & 13.50 \\
\hline 'NM94' & 11.59 & 1.19 & 0.52 & 9.77 \\
\hline 'EG-MD-6D' & 16.44 & 1.40 & 0.72 & 13.82 \\
\hline 'CES55' & 14.32 & 1.32 & 0.66 & 12.22 \\
\hline 'MG50-10A (Y)' & 12.58 & 1.59 & 0.60 & 7.72 \\
\hline $\begin{array}{c}\text { 'BPI GLABROUS } \\
\# 3 '\end{array}$ & 17.45 & 1.51 & 0.87 & 13.47 \\
\hline 'WALET' & 14.80 & 1.18 & 0.77 & 13.14 \\
\hline ‘GELATIK’ & 15.68 & 1.51 & 0.95 & 10.71 \\
\hline 'KING' & 12.03 & 1.59 & 0.71 & 8.60 \\
\hline 'TAINAN SEL\#5’ & 15.48 & 1.52 & 0.75 & 10.27 \\
\hline 'CN2' & 9.95 & 1.01 & 0.36 & 10.54 \\
\hline 'CN80' & 8.92 & 0.98 & 0.39 & 9.31 \\
\hline 'BR-1' & 8.28 & 1.21 & 0.44 & 8.01 \\
\hline 'PAK40592' & 9.92 & 0.96 & 0.33 & 10.93 \\
\hline
\end{tabular}

Notes: $\mathrm{Pn}=$ net photosynthetic rate; $\mathrm{Tr}=$ transpiration rate; $\mathrm{Gs}=$ stomatal conductance; $\mathrm{WUE}=$ water use efficiency.

We also found strong positive correlations between clusters/plant with branches/plant and pods/plant. This finding is not surprising because the pods increased, together with the clusters, which are in turn formed by the branches. Note that clusters/plant, branches/plant, and pods/plant were strongly negatively related to pod length, pod width, and 100-seed weight. In this study, the mungbean genotypes, i.e. 'ML-131', 'VAR A-G', and 'BARI MUNG2', possessed higher clusters/plant, branches/plant, pods/plant and lower pod length, pod width, and 100-seed weight. Moreover, there were significant positive correlations between plant height with pod length, pod width, seeds/pod, Pn, Gs, and WUE and significant negative correlation with branches/plant. The genotypes, i.e. 'CN36' and 'EG-MD-6D', appeared to have taller plant, higher pod length, pod width, seeds/pod, Pn and lower branches/plant.

The average pair-wise Euclidean distance of the phenogram among all genotypes based on all the agronomic and photosynthetic variables and disease responses was 5.483, ranging from 1.015 ('NM92' vs. 'WALET') to 9.386 ('SUT1' vs. 'PAK40592'). The cophenetic correlation coefficient value of 0.7836 ( $\mathrm{p}<$ 0.01 ) was observed, demonstrating a high association for these genotypes to a specific cluster represented in the dendrogram (Figure 1A). However, bootstrap analysis revealed high values only for cluster I, IV and V, and lower values in the remaining nodes, indicating a lack of robustness of the clustering in some clusters. A low support value can be obtained if genotypes cover an intermediate position between major groups (García- 
Martínez et al., 2006). All blackgram genotypes were grouped in cluster I. Several mungbean genotypes formed four clusters including cluster II, III, IV and V consisting of five, nine, five, and two genotypes, respectively.

Table 6. Pearson correlation coefficients of agronomic and photosynthetic characters of 23 mungbean and four blackgram genotypes

\begin{tabular}{|c|c|c|c|c|c|c|c|c|c|c|c|c|c|c|c|}
\hline $\begin{array}{c}\text { Characte } \\
\text { rs } \\
\end{array}$ & YPP & DTF & DTM & СРP & BPP & PH & PPP & PL & PW & HSW & SPP & Pn & $\operatorname{Tr}$ & Gs & WUE \\
\hline YPP & & $0.200^{\circ}$ & $0.029^{\mathrm{ns}}$ & $0.169^{\text {ns }}$ & $0.091^{\mathrm{ns}}$ & $0.569^{\prime \prime}$ & $0.435^{\prime \prime}$ & $0.430^{\prime \prime}$ & $0.041^{\mathrm{ns}}$ & $0.045^{\text {ns }}$ & $0.612^{\prime \prime}$ & $0.463^{\prime \prime}$ & $0.210^{\circ}$ & $0.406^{\prime \prime}$ & $0.235^{\circ}$ \\
\hline DTF & & & $0.714 "$ & $0.134^{\mathrm{ns}}$ & $0.038^{\mathrm{ns}}$ & $0.463^{\prime \prime}$ & $0 . \overline{170^{\text {ns }}}$ & $0.290^{\prime \prime}$ & $0.188^{\text {ns }}$ & $0.147^{\mathrm{ns}}$ & $0.296^{\prime \prime}$ & $0.194^{\mathrm{ns}}$ & 0.137 & $0.290^{\prime \prime}$ & $0.105^{\text {ns }}$ \\
\hline DTM & & & & $0.000^{\mathrm{ns}}$ & $0.282^{\prime \prime}$ & $0.221^{\circ}$ & $-0.264^{\prime \prime}$ & $0.005^{\mathrm{ns}}$ & $0.160^{\mathrm{ns}}$ & $0.195^{\text {ns }}$ & $\overline{-}_{0.126^{\mathrm{ns}}}$ & $0.048^{\mathrm{ns}}$ & $0.060^{\text {ns }}$ & $0.156^{\mathrm{ns}}$ & $0.093^{\text {ns }}$ \\
\hline СРP & & & & & $0.781^{\prime \prime}$ & $\begin{array}{c}- \\
0.006^{\mathrm{ns}}\end{array}$ & $0.769^{\prime \prime}$ & $-0.639^{\prime \prime}$ & $-0.705^{\prime \prime}$ & $-0.679^{\prime \prime}$ & $\begin{array}{c}- \\
0.155^{\mathrm{ns}} \\
\end{array}$ & $\begin{array}{c}- \\
0.019^{\mathrm{ns}} \\
\end{array}$ & $0 . \overline{-}$ & $\begin{array}{c}- \\
0.055^{\mathrm{ns}} \\
\end{array}$ & $0.027^{\text {ns }}$ \\
\hline BPP & & & & & & $-0.226^{\circ}$ & $0.542^{*}$ & $-0.757^{\prime \prime}$ & $-0.694^{\prime \prime}$ & $-0.634^{\prime \prime}$ & $-0.367^{\prime \prime}$ & $0.113^{\text {ns }}$ & $\begin{array}{c}- \\
0.126^{\mathrm{ns}}\end{array}$ & $\begin{array}{c}- \\
0.066^{\mathrm{ns}}\end{array}$ & $0.037^{\text {ns }}$ \\
\hline $\mathrm{PH}$ & & & & & & & $0.004^{\mathrm{ns}}$ & $0.481^{\prime \prime}$ & 0.206 & $0.117^{\mathrm{ns}}$ & $0.610^{\circ \prime}$ & $0.592^{\prime \prime}$ & $0.034^{\text {ns }}$ & $0.530^{\prime \prime}$ & $0.498 "$ \\
\hline PPP & & & & & & & & $-0.464^{\prime \prime}$ & $-0.713^{\prime \prime}$ & $-0.754^{\prime \prime}$ & $0.108^{\mathrm{ns}}$ & $0.128^{\mathrm{ns}}$ & $0.059^{\mathrm{ns}}$ & $0.074^{\mathrm{ns}}$ & $0.046^{\mathrm{ns}}$ \\
\hline PL & & & & & & & & & $0.750^{\prime \prime}$ & $0.662^{\prime \prime}$ & $0.615^{\prime \prime}$ & $0.340^{\prime \prime}$ & $0.237^{\circ}$ & $0.274^{\prime \prime}$ & $0.151^{n s}$ \\
\hline PW & & & & & & & & & & $0.941^{\prime \prime}$ & $0.031^{\mathrm{ns}}$ & $0.038^{\mathrm{ns}}$ & $0.105^{\text {ns }}$ & $0.069^{\mathrm{ns}}$ & $0.021^{\text {ns }}$ \\
\hline HSW & & & & & & & & & & & $0.125^{\mathrm{ns}}$ & $0.000^{\mathrm{ns}}$ & $0.052^{\text {ns }}$ & $0.006^{\mathrm{ns}}$ & $0.018^{\text {ns }}$ \\
\hline SPP & & & & & & & & & & & & $0.472^{* *}$ & $0.214^{\circ}$ & $0.392^{\prime \prime}$ & $0.264^{\prime \prime}$ \\
\hline Pn & & & & & & & & & & & & & $0.151^{\mathrm{ns}}$ & $0.748^{\prime \prime}$ & $0.712 "$ \\
\hline $\mathrm{Tr}$ & & & & & & & & & & & & & & $0.188^{\mathrm{ns}}$ & $-0.504^{\prime \prime}$ \\
\hline Gs & & & & & & & & & & & & & & & $0.496^{\prime \prime}$ \\
\hline
\end{tabular}

Notes: YPP $=$ Yield/plant; DTF $=$ Days to flowering; DTM = Days to maturity; CPP = Clusters/plant; BPP = branches/plant; PH = Plant height; PPP = Pods/plant; PL = Pod length; PW = Pod width; HSW = 100 seed-weight; $\mathrm{SPP}=$ Seeds/pod; $\mathrm{Pn}=$ net photosynthetic rate; $\mathrm{Tr}=$ transpiration rate; Gs = stomatal conductance; WUE = water use efficiency." significant correlation at the 0.01 level (two-tailed). " significant correlation at the 0.05 level (twotailed).

In cluster II, 'V4718', 'V4758', and 'V4785', originated in India whose genetics are related to disease resistance in Thailand (Poolsawat et al., 2017; Tantasawat et al., 2020; Tantasawat unpublished data) showed high resistance to CLS and PM (Table 4). However, the others failed in their resistance to both diseases, although some of them, i.e. 'PUSA-105', which also originated in India was found to have high resistance to CLS in India (Marappa, 2008), indicating the influence of race specific resistance. Cluster III and IV consisting of several mungbean representatives from different regions including Thailand, the Philippines, Australia, Pakistan, Taiwan, and Indonesia are interesting, as members in both clusters shared similar characters, particularly pod width, pod length, and 100-seed weight (Table 7). However, the average plant height of cluster IV was higher than that of cluster III which may separate them into two distinct clusters. Note that several members, i.e. 'NM92', 'CES55', 'WALET', and 'TAINAN SEL\#5' in cluster III and 'CN36', 'CN84-1', and 'SUT1' in cluster IV also produced high yield/plant. Some of these varieties which originated in other countries, i.e. 'WALET', the local high yielding variety of Indonesia (Hakim, 2008) also had high yielding potential when grown in Thailand. Moreover, Thai certified varieties including 'CN36', 'KPS1' and 'SUT1' in cluster IV, as well as 'SUT4' in cluster III, which were reported to have resistance to CLS and PM were susceptible to both diseases in this study. This may be attributed to their resistance breakdown by virulent pathogens. Two mungbean genotypes including 'ML-131' and 'BARI-MUNG 2' showing high pods/plant and high yield/plant were grouped into cluster V. In previous studies, cluster analysis based on the characters related to yield, i.e. seed yield/plant, biological yield, and harvest index was not allocated in different clusters/subclusters because they characterized a large number of mungbean accessions, i.e. 340 and 533 accessions in Yimram et al. (2009) and Tahir et al. (2020), respectively. While NM94 and VAR A-G were individuals, not grouped into any clusters. The PCoA revealed that the utmost total variation of $49 \%$ from the sum of $89 \%$ was largely explained by the first principal coordinate, and $40 \%$ was from coordinate two. The clustering pattern of the PCoA was similar to the dendrogram generated by UPGMA analysis. However, most mungbean genotypes on the PCoA axis were not well distinguished as seen in the dendrogram (Figure 1B). 


\section{SRAP analysis and genetic relationships}

A set of 25 SRAP primer combinations revealed a high polymorphism percentage of $90.2 \%$ (representing the profiles of 507 polymorphic bands from 562 scorable bands with a size range of approximately 200 to 1,400 bp as shown in Table 3). However, Aneja et al. (2013) and Alghamdi et al. (2017) found 100\% polymorphism among Indian mungbean varieties and Saudi mungbean germplasms, respectively. Although we obtained a comparatively lower polymorphism percentage than those of previous studies, our study used higher numbers of primer combinations and scorable DNA bands, both of which may affect polymorphism percentage. We used silver staining on polyacrylamide gel, which detected higher number of scorable bands than ethidium bromide staining on agarose gel as used in previous study (Aneja et al., 2013). These results indicate that polyacrylamide gel with smaller pore sizes and higher resolution than agarose gel can effectively visualize and differentiate small DNA bands, mostly obtained from multilocus marker systems (Bassam and Gresshoff, 2007; Tantasawat et al., 2010). Each primer combination generated an average of 22 bands, of which 20 exhibited polymorphism. The lowest number of polymorphic bands was achieved with the primer combination me2em3 (7) with polymorphism percentage of 50 . Whereas, the primer combinations melem 2 generated the highest numbers of polymorphic bands (38). This primer combination together with me3em1 (21), me3em2 (20), me3em4 (22), me4em3 (19), me4em4 (21), and me4em5 (16) had the highest polymorphism percentage of 100. From all primer combinations, an average polymorphism percentage of $89 \%$ was revealed across all genotypes. This was comparable to that found in mungbean and blackgram (89.51\%) using inter-simple sequence repeat (ISSR) markers (Tantasawat et al., 2010). However, the polymorphism levels may be restricted by the self-pollinated nature of these two species.

The PIC values for each primer are most often analyzed to estimate discriminatory power by accounting both the number of alleles at a locus and their relative frequencies (Nagl et al., 2011), and it can reach a maximum of 0.5 (in case of dominant markers). The PIC values of $\geq 0.28$ in eight primer combinations; melem2, me2em2, me3em3, me4em1, me $4 \mathrm{em} 3$, me4em4, me $5 \mathrm{em} 3$, and me $5 \mathrm{em} 5$, and 100\% polymorphism in three of these primer combinations; melem 2 , me $4 \mathrm{em} 3$, and me4em 4 suggested that they are preferable for genetic characterization. These results demonstrate that using only eight primer combinations, including melem2, me2em2, me3em 3 , me4em 1, me4em3, me $4 \mathrm{em} 4$, me $5 \mathrm{em} 3$, and me $5 \mathrm{em} 5$ allows one to distinguish the genotypes evaluated within and between both species. Therefore, the use of this marker system can reduce cost, time, and labor in the identification of varieties of mungbean and blackgram. Tantasawat et al. (2010) obtained similar findings when using only six most informative ISSR primers. However, genetic identification based on SRAP loci is more likely to reflect phenotypic trait expressions than other multilocus markers including ISSR markers, which are largely located in non-coding genomic regions (Shao et al., 2010).

Pair-wise Euclidean distance coefficient values varied from 4.107 ('CN36' vs. 'CN72') to 57.846 ('V4758' vs. 'BR-1') with an average of 26.447 . Average distance coefficient between $V$. radiata genotypes was 15.776. 'CN36' and 'CN72' which are widely used commercially in Thailand and possess some relationship in their pedigree (Table 1) showed the lowest Euclidean distance (4.107), while 'V4758' and 'CES55' without any relationship showed the highest Euclidean distance (21.566) in the mungbean group. 'CN36', 'CN72', and 'CES55' had high pod length, pod width, and 100-seed weight, whereas 'V4758' did not. In the blackgram group, the genetic distances ranged from 24.749 to 28.734 found between 'CN2' with 'CN80' and between 'CN80' with 'PAK40592', respectively, with an average of 27.120. Phylogenetically genotypic data between both species revealed their genetic distance of 55.749, indicating a high interspecific diversity that reflects the differences observed in their several agronomic and photosynthetic characters, i.e. plant height, pod length, seeds/pod, yield/plant, Pn, Tr, and Gs. The SRAP-based UPGMA dendrogram showed two distinct clusters with $100 \%$ bootstrap value where all four blackgram genotypes were representatives of cluster I, while cluster II comprised all 23 mungbean genotypes (Figure 2A). The coefficient cophenetic value with Mantel's test reached $0.9959(\mathrm{p}<0.01)$, indicating high reliability of distance matrix data represented in the dendrogram. 
A
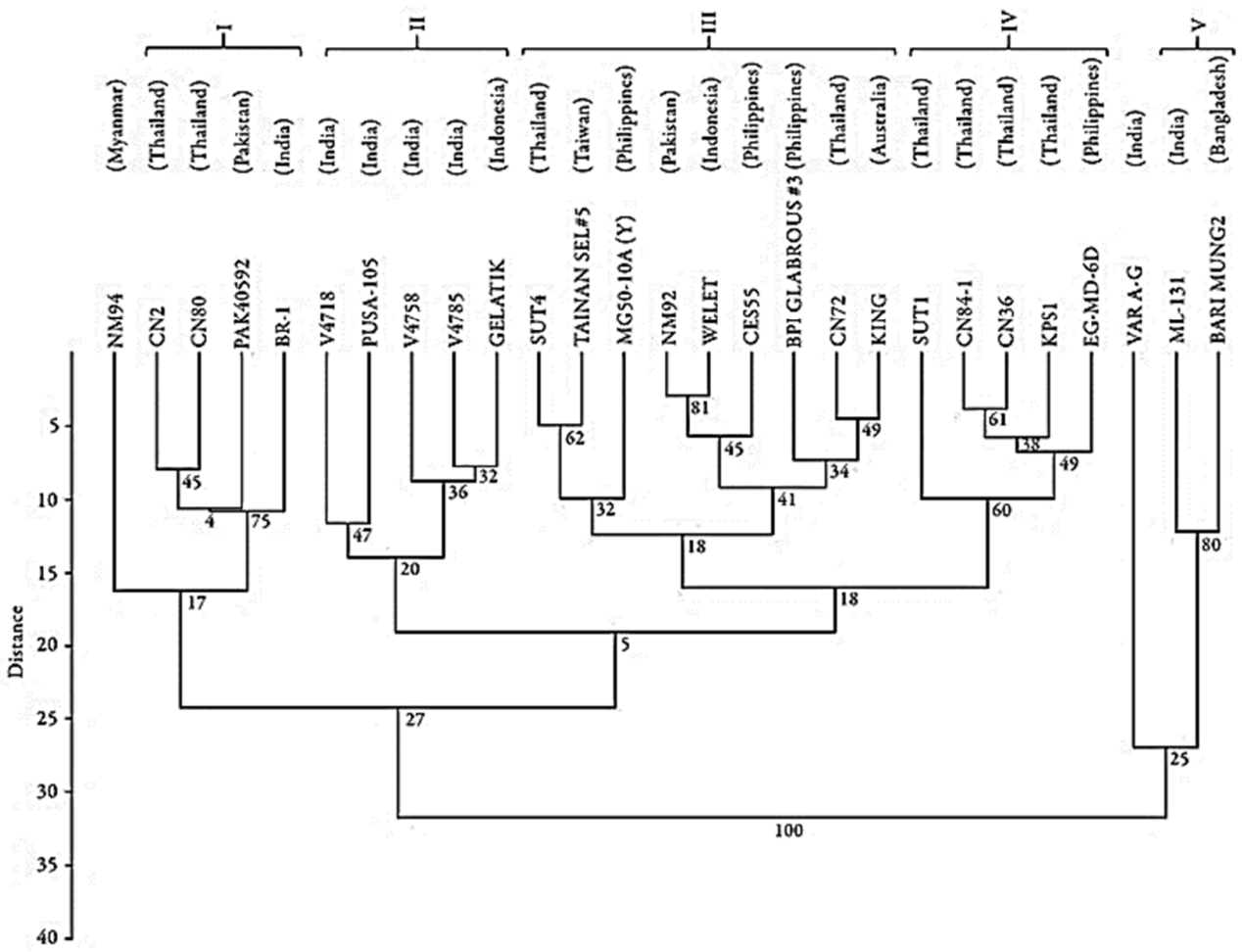

B

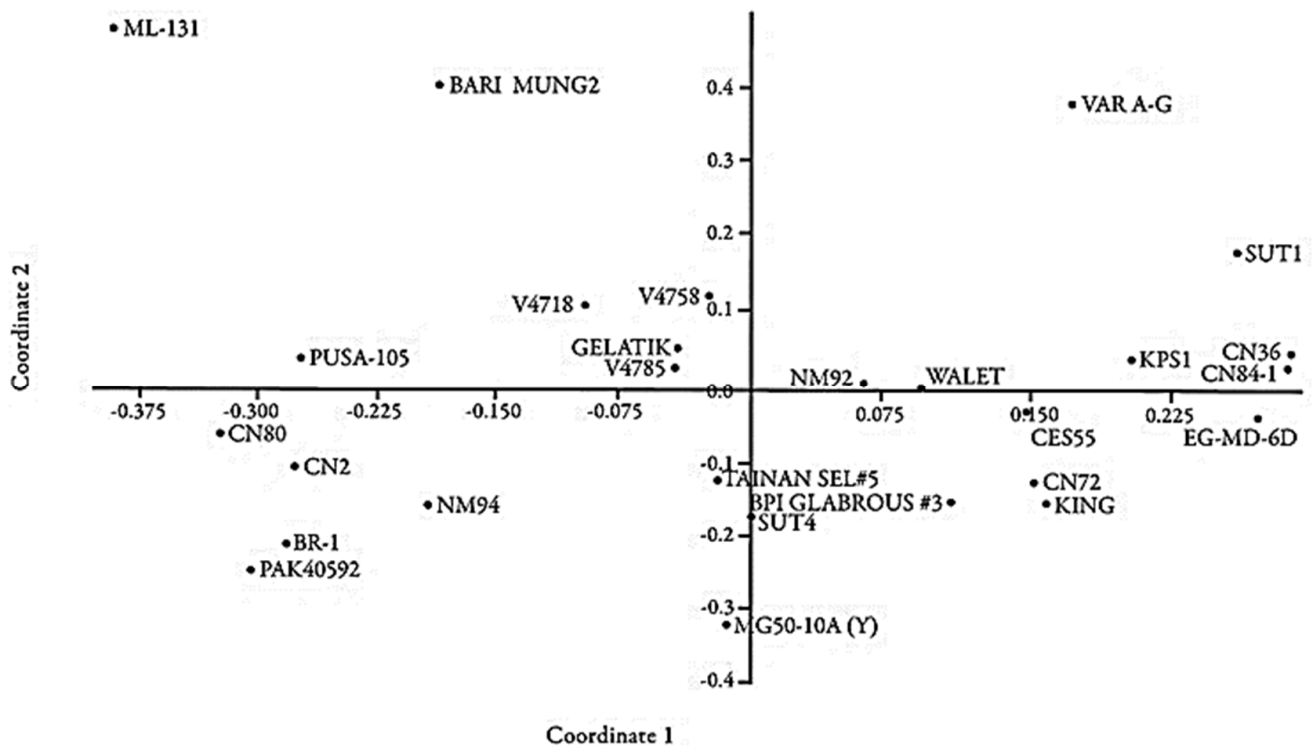

Figure 1. A dendrogram showing the agronomic and photosynthetic characters generated by an unweighted pair group method average (UPGMA) (A) and a two-dimensional plot based on the first two principal coordinates from the principal coordinate analysis (PCoA) (B) of 23 mungbean and four blackgram genotypes 
Table 7. Means of agronomic and photosynthetic characters of 23 mungbean and four blackgram genotypes in different clusters

\begin{tabular}{|c|c|c|c|c|c|c|c|}
\hline \multirow{2}{*}{ Characters } & \multicolumn{5}{|c|}{ Clusters (number of genotypes) } & \multicolumn{2}{|c|}{ Individuals } \\
\hline & $\mathrm{I}(4)$ & II $(5)$ & III (9) & $\mathrm{IV}(5)$ & $\mathrm{V}(2)$ & NM94 & VAR A-G \\
\hline Days to flowering & 36.09 & 38.20 & 38.88 & 39.80 & 36.92 & 31.50 & 40.50 \\
\hline Days to maturity & 55.75 & 53.15 & 54.26 & 54.90 & 51.63 & 46.50 & 54.25 \\
\hline Clusters/plant & 12.21 & 9.04 & 6.25 & 6.82 & 14.59 & 6.75 & 14.96 \\
\hline Branches/plant & 3.05 & 1.78 & 0.66 & 0.82 & 2.53 & 0.45 & 2.13 \\
\hline Plant height $(\mathrm{cm})$ & 40.09 & 53.12 & 55.54 & 69.80 & 54.89 & 43.31 & 75.24 \\
\hline Pods/plant & 25.37 & 30.82 & 18.00 & 19.84 & 52.07 & 23.20 & 35.57 \\
\hline Pod length $(\mathrm{cm})$ & 4.64 & 7.39 & 9.68 & 9.59 & 6.88 & 9.49 & 7.83 \\
\hline Pod width $(\mathrm{mm})$ & 5.18 & 5.03 & 6.36 & 6.43 & 4.80 & 5.79 & 4.66 \\
\hline 100-seed weight $(\mathrm{g})$ & 5.20 & 3.93 & 7.05 & 7.01 & 3.41 & 5.68 & 3.35 \\
\hline Seeds/pod & 5.59 & 10.44 & 9.79 & 9.97 & 10.00 & 10.19 & 11.80 \\
\hline Yield/plant (g) & 6.03 & 9.13 & 9.16 & 10.18 & 11.37 & 9.34 & 10.69 \\
\hline $\operatorname{Pn}\left(\mu \mathrm{mol} \mathrm{m} \mathrm{m}^{-2} \mathrm{~s}^{-1}\right)$ & 9.27 & 14.24 & 14.45 & 15.38 & 15.98 & 11.59 & 14.09 \\
\hline $\operatorname{Tr}\left(\mathrm{mmol} \mathrm{m} \mathrm{m}^{-2} \mathrm{~s}^{-1}\right)$ & 1.04 & 1.44 & 1.39 & 1.43 & 1.54 & 1.19 & 1.35 \\
\hline $\mathrm{Gs}\left(\mathrm{mol} \mathrm{m} \mathrm{s}^{-1}\right)$ & 0.38 & 0.77 & 0.73 & 0.82 & 0.79 & 0.52 & 0.63 \\
\hline WUE $\left(\mathrm{mmol} \mathrm{mol}^{-1}\right)$ & 9.70 & 11.03 & 11.30 & 11.92 & 10.84 & 9.77 & 10.66 \\
\hline Cercospora leaf response & 1.75 & 3.47 & 4.74 & 4.67 & 5.00 & 5.00 & 4.00 \\
\hline $\begin{array}{l}\text { Powdery mildew } \\
\text { response }\end{array}$ & 7.17 & 4.33 & 6.33 & 6.60 & 7.50 & 7.33 & 8.33 \\
\hline
\end{tabular}

In cluster II, there were two subclusters largely consistent with their special features reported between high yielding potential (subcluster IIA) and resistance to diseases (subcluster IIB) (Table 1). These results support our previous report, which used 11 expressed sequence tags-simple sequence repeat (EST-SSR) primer pairs to determine the genetic diversity and relationships of these 27 genotypes (Chueakhunthod et al., 2018). SRAP-based clustering was apparently clearer than that of the EST-SSR system because 11 of those 18 genotypes reported to have resistance to diseases including 'PUSA-105', 'VAR A-G', 'NM92', 'TAINAN SEL\#5', 'GELATIK', 'BARI MUNG2', 'NM94', 'ML-131', 'V4718', 'V4758', 'V4785' were separated from the others reported to have high yielding potential, while only five of these were grouped together by EST-SSR markers. This may be due to the higher number of polymorphic bands derived from SRAP ( 507 bands) compared to EST-SSR ( 56 bands). All the genotypes which originated from India with resistance to diseases were grouped in subcluster IIB, separated from subcluster IIA where the genotypes from Thailand and the Philippines as well as Indonesia and Australia with high yielding potential were grouped together. There were 12.909 and 10.886 genetic distances among genotypes from the Philippines and Thailand, respectively, which were lower than those from India (17.318 genetic distance in subcluster IIB). In the case of the Philippines individual similarity, this can be explained by the fact that the Philippines improved varieties or lines were initially developed from only six purified local varieties as parents (Ballon et al., 1978; Catipon et al., 1988; Somta et al., 2009). Several outstanding Philippines varieties/lines developed were intensively used as parents both nationally and internationally (Catipon et al., 1988), and this may account for the sharing of common parents and a narrow genetic base. For example, MG50-10A obtained from crossing between 'GLOSSY GREEN S1' and 'GLABROUS GREEN' was utilized to develop 'CES55' and 'BPI GLABROUS \#3'. Somta et al. (2009) also revealed this close relationship among the Philippines mungbeans when using SSR markers. Thai mungbean genotypes in this study also shared common parents in their pedigree. For example, 'CN84-1' was derived from the $\gamma$ irradiated mutants of 'CN36', or 'CN72' was selected from mutated 'KPS2', which contains genetic background of ' $\mathrm{CN} 36$ ' (Table 1). On the other hand, three Indian mungbean genotypes in 
subcluster IIB; 'V4718', 'V4758', and 'V4785', whose CLS and PM resistance genes were identified by Chankaew et al. (2011); Poolsawat et al. (2017) and Tantasawat (unpublished data), and found to be highly resistant to both diseases (Table 4) had genetic distance of 14.216. In subcluster IIA, 'SUT1', 'CN36', 'CN841', and 'EG-MD-6D' which had high yielding potential with regard to high yield/plant and large seed size and were grouped in the same subcluster IV of the dendrogram based on field performance (Figure 1A) exhibited high genetic relationships with only 10.729 genetic distance. To understand the genetic relationships among the genotypes better, the PCA was also performed. Two principal coordinates explained $77 \%$ of the total variation, of which the first two axes accounted for 68 and $9 \%$, respectively. Similar to UPGMA analysis, this method showed two distinct groups according to species similar to the cluster analysis (Figure 2B).

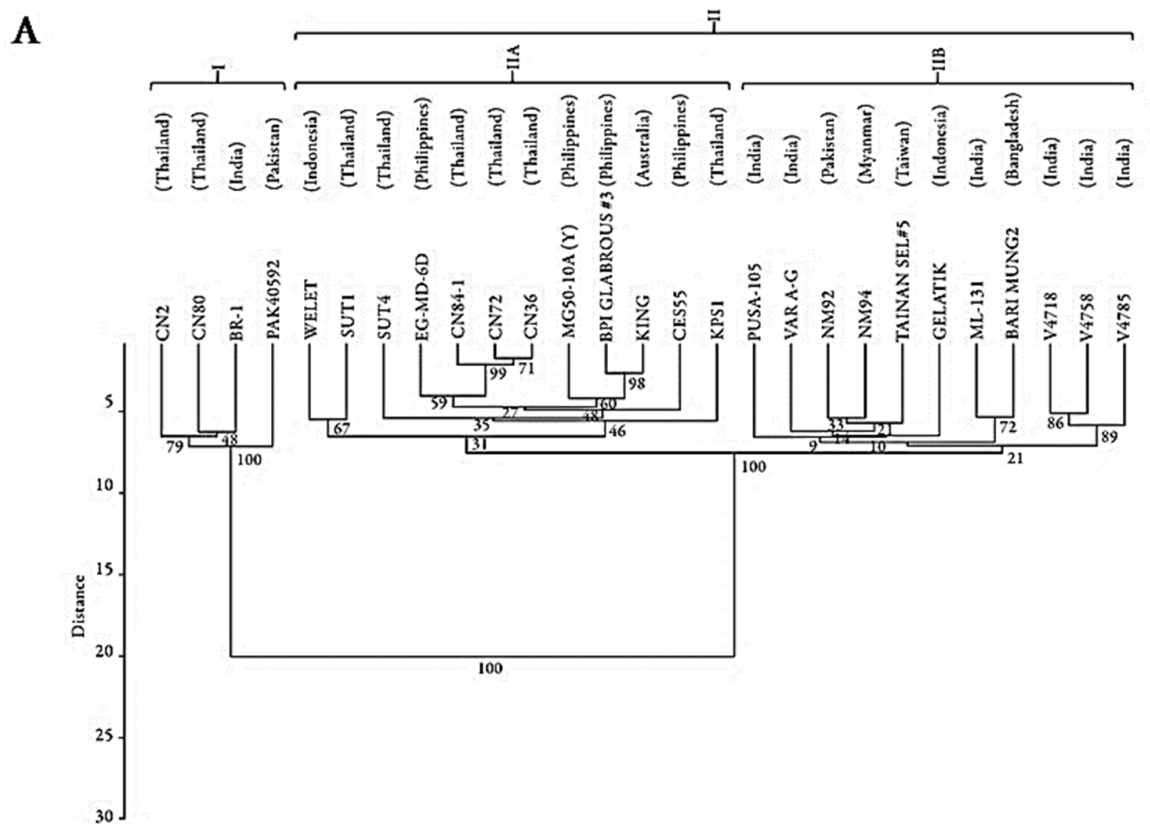

\section{B}

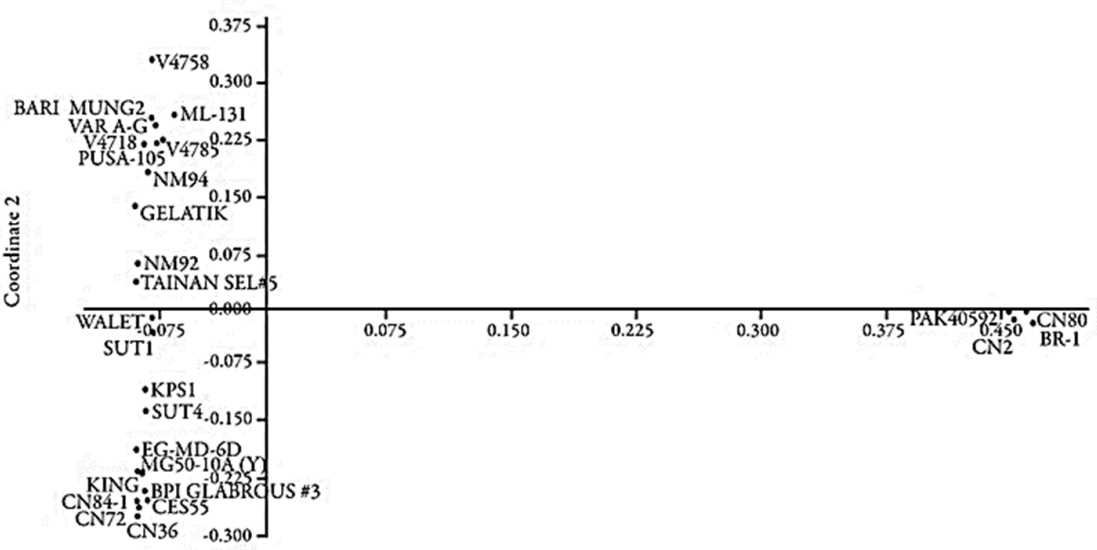

Coordinate 1

Figure 2. A dendrogram showing the SRAP markers generated by an unweighted pair group method average (UPGMA) (A) and a two-dimensional plot based on the first two principal coordinates from the principal coordinate analysis (PCoA) $(\mathrm{B})$ of 23 mungbean and four blackgram genotypes 


\section{Comparison between marker systems}

SRAPs implicate a greater influence of the characters due to their exclusive strategies, which are proved with an association of $0.734(\mathrm{p}<0.01)$ between this functional marker and the characters related to field performance, indicating a concordance between both marker systems. This finding was consistent with Aneja et al. (2013), who stated that the information given by SRAP enabled one to classify several mungbean genotypes on the basis of micronutrient content. Ferriol et al. (2003) also reported that SRAP corresponded more closely to morphological characters and to the evolutionary history by means of the morphotypes than amplified fragment length polymorphism (AFLP). This coherence between SRAP and morphological pattern has also been reported in other legume crops, i.e. pea and chickpea (Espósito et al., 2009; Khan et al., 2016). The mungbean and blackgram genotypes were largely grouped into separate clusters according to species by each marker system. However, some variations in the clustering from different marker systems should also be noted (Figures $1 \mathrm{~A}$ and 2A). SRAPs proved to be more effective than agronomic and photosynthetic characters in unravelling genetic differentiation between these $V$. radiata and $V$. mungo genotypes, since the bootstrap value of the $V$. radiata and $V$. mungo clusters was $100 \%$. In addition, cophenetic correlations for testing the goodness of fit from SRAPs were higher than those of the agronomic and photosynthetic characters $(0.9959$ vs. 0.7836). Moreover, the SRAP marker loci were capable of classifying most mungbean genotypes according to their special features between disease resistance and high yielding potential.

\section{Conclusions}

These results demonstrate that the multilocus SRAP marker system with apparent strategies in functional analysis mediated by the amplification of DNA using a single forward primer with numerous interchangeable reverse primers has proved to be a valuable tool in determining variability and the relationships among these two Vigna species and in trait mapping or marker-assisted selection. The promising mungbean genotypes, including Thai certified varieties, i.e. 'SUT1', 'CN84-1', and 'CN36' and those from other regions, i.e. 'EG-MD-6D' from the Philippines possessed characters, i.e. pod length, pod width, 100-seed weight, yield/plant, and photosynthetic characters that are very useful for improving yield. All of these candidates can contribute to future breeding programs, while the others, i.e. 'V4718', 'V4758', and 'V4785', which contain resistance genes enabling them to have high resistance to CLS and PM can also be used as parents in a gene pyramiding program for durable disease resistance. In addition, seeds/pod, plant height, pods/plant, pod length, days to flowering, and all photosynthetic characters studied including Pn, Gs, WUE, and Tr, which contributed the most to yield/plant can be used as selection criteria for yield improvement in both mungbean and blackgram.

\section{Authors' Contributions}

Conceptualization: PAT; Materials: SN; Methodology: WC, WJ, and PAT; Validation: WJ and PAT; Formal analysis: WC; Investigation: WC, KM, RK, PP, NJ, and PP; Data curation: WC, WJ, and PAT; Funding acquisition: PAT; Project administration: PAT; Writing: WC and PAT; Review and editing; WJ and PAT. All authors read and approved the final manuscript.

\section{Acknowledgements}


This work was supported by SUT Research and Development Fund. The World Vegetable Center, Taiwan and Chai Nat Field Crops Research Center, Thailand are gratefully acknowledged for providing the mungbean and blackgram seeds for the current study. The authors are very grateful to Peter Charles Bint and Jason Biscombe for editing the manuscript.

\section{Conflict of Interests}

The authors declare that there are no conflicts of interest related to this article.

\section{References}

Alghamdi SS, Al-Faifi SA, Migdadi HM, Al-Rowaily SL, El-Harty EH, Farooq M (2017). Genetic diversity and field performance of mung bean, faba bean and lentil genotypes in the kingdom of Saudi Arabia. International Journal of Agriculture and Biology 19:689-696. https://doi.org/10.17957/IJAB/15.0336

Aneja B, Yadav NR, Yadav RC, Kumar R (2013). Sequence related amplified polymorphism (SRAP) analysis for genetic diversity and micronutrient content among gene pools in mungbean [Vigna radiata (L.) Wilczek]. Physiology and Molecular Biology of Plants 19:399-407. https://doi.org/10.1007/s12298-013-0177-3

Ballon FB, Legaspi BM, Catipon EM (1978). Mungbean varietal improvement program of the bureau of plant industry. In: Cowell R (Ed). The First International Mungbean Symposium. AVRDC, Shanhua, Taiwan pp 217-219.

Bassam BJ, Gresshoff PM (2007). Silver staining DNA in polyacrylamide gels. Nature Protocols 2:2649-2654. https://doi.org/10.1038/nprot.2007.330

Catipon EM, Legaspi BM, Jarilla FA (1988). Development of mungbean varieties from AVRDC lines for the Philippines. In: Shanmugasundaram S, Mclean BT (Eds). Mungbean: Proceedings of the Second International Symposium. AVRDC, Shanhua, Taiwan pp 88-97.

Chai Nat Field Crops Research Center (2018). Guidelines for data record in mungbean research. Chai Nat Field Crops Research Center, Field and Renewable Energy Crops Research Institute, Department of Agriculture pp 49-54.

Chankaew S, Somta P, Sorajjapinun W, Srinives P (2011). Quantitative trait loci mapping of Cercospora leaf spot resistance in mungbean, Vigna radiata (L.) Wilczek. Molecular Breeding 28:255-264. https://doi.org/10.1007/s11032-010-9478

Chueakhunthod W, Ngampongsai S, Jinagool W, Tantasawat PA (2018). Genetic diversity of mungbean and blackgram based on EST-SSR analysis. International Journal of Advances in Science, Engineering and Technology 6:78-82. http://ijaseat.iraj.in/paper_detail.php?paper_id=121868name=Genetic_Diversity_of_Mungbean_and_Blac kgram_based_on_EST-SSR_Analysis

Curtis PE, Ogreen WL, Hagmen RH (1969). Varietal effect in soybean photosynthesis and photorespiration. Crop Science 10:42-45. https://doi.org/10.2135/cropsci1969.0011183X000900030021X

Das RT, Barua PK (2015). Association studies for yield and its components in green gram. International Journal of Agriculture Environment and Biotechnology 8:561-565. https://doi.org/10.5958/2230-732X.2015.00086.8

Espósito MA, Milanesi LA, Martin E, Cointry E (2009). Comparison of morphological and molecular data for pea (Pisum sativum) in low and high yielding environments. New Zealand Journal of Crop and Horticultural Science 37:227-233. https://doi.org/10.1080/01140670909510268

Ferriol M, Picó B, Nuez F (2003). Genetic diversity of a germplasm collection of Cucurbita pepo using SRAP and AFLP. Theoretical and Applied Genetics 107:271-282. https://doi.org/10.1007/s00122-003-1242-z

Gao J, Seneweera S, Li P, Zong YZ, Dong Q, Lin E, Hao X (2015). Leaf photosynthesis and yield components of mung bean under fully open-air elevated $\left[\mathrm{CO}_{2}\right]$. Journal of Integrative Agriculture 14:977-983. https://doi.org/10.1016/S2095-3119(14)60941-2

García-Martínez S, Andreani L, Garcia-Gusano M, Geuna F, Ruiz JJ (2006). Evaluation of amplified fragment length polymorphism and simple sequence repeats for tomato germplasm fingerprinting: utility for grouping closely related traditional cultivars. Genome 49:648-656. https://doi.org/10.1139/g06-016 
Garg GK, Verma PK, Kesh H (2017). Genetic variability, correlation and path analysis in mungbean [Vigna radiata (L.) Wilczek]. International Journal of Current Microbiology and Applied Sciences 6:2166-2173. https://doi.org/10.20546/ijcmas.2017.611.255

Hakim L (2008). Variability and correlation of agronomic characters of mungbean germplasm and their utilization for variety improvement program. Indonesian Journal of Agricultural Science 9:24-28. https://doi.org/10.21082/ijas.v9n1.2008.p24-28

Hemavathy AT, Shunmugavalli N, Anand G (2015). Genetic variability, correlation and path co-efficient studies on yield and its components in mungbean [Vigna radiata (L.) Wilezek]. Legume Research 38:442-446. https://doi.org/10.5958/0976-0571.2015.00050.8

Hossain MA, Hamid A, Khaliq MA (2009). Evaluation of mungbean (Vigna radiata (L.) Wilczek) genotypes on the basis of photosynthesis and dry matter accumulation. Journal of Agriculture \& Rural Development 7:1-8. https://doi.org/10.3329/jard.v7i1.4415

IBPGR Secretariat (1980). Descriptor for mung bean. AGP:IBPGR/80/35, Rome pp 1-15.

Islam MT, Agata W, Kubota F, Hamid A (1994). Evaluation of characteristics related to photosynthetic rate and leaf area production in mungbean genotypes. Japanese Journal of Crop Science 63:502-509.

Islam MT, Razzaque AHM (2010). Relationships of photosynthetic and related parameters and yield of summer mungbean varieties/mutants. International Journal of Sustainable Crop Production 5:11-15. https://docplayernet/145082033-Relationships-of-photosynthetic-related-parameters-and-yield-of-summermungbean-varieties-mutants.html

Khan MA, Ammar MH, Migdadi HM, El-Harty EH, Alfaifi SA, Farooq M, Alghamdi SS (2016). Field performance and genetic diversity of chickpea genotypes. International Journal of Agriculture and Biology 18:683-688. https://doi.org/10.17957/IJAB/15.0151

Khajudparn P (2009). Characters associated with yield potential and development of molecular markers for powdery mildew resistance in mungbean. PhD Thesis, Suranaree University of Technology, Nakhon Ratchasima.

Khajudparn P, Tantasawat P (2011). Relationships and variability of agronomic and physiological characters in mungbean. African Journal of Biotechnology 10:9992-10000. https://doi.org/10.5897/AJB11.1288

Khajudparn P, Wongkaew S, Thipyapong P (2007). Mungbean powdery mildew resistance. Identification of genes for powdery mildew resistance in mungbean. In: Proceeding of the 8th African Crop Science Conference. El-Minia, Egypt pp 743-745.

Khattak GSS, Srinives P, Kim DH (1995). Yield partitioning in high yielding mungbean (Vigna radiata (L.) Wilczek). Kasetsart Journal: Natural Science 29:494-497. https://www.lib.ku.ac.th/KUJN/TAB451304.pdf

Kumar A, Sharma NK, Kumar R, Sanadya SK, Sahoo S (2018). Correlation and path analysis for seed yield and components traits in mungbean under arid environment. International Journal of Chemical Studies 6:16791681.

Lapointe FJ, Legendre P (1992). Statistical significance of the matrix correlation coefficient for comparing independent phylogenetic trees. Systematic Biology 41:378-384. https://doi.org/10.1093/sysbio/41.3.378

Li G, Quiros CF (2001). Sequence-related amplified polymorphism (SRAP), a new marker system based on a simple PCR reaction: its application to mapping and gene tagging in Brassica. Theoretical and Applied Genetics 103:455461. https://doi.org/10.1007/s001220100570

Lodhi MA, Ye GN, Weeden NF, Reisch BI (1994). A simple and efficient method for DNA extraction from grapevine cultivars and Vitis species. Plant Molecular Biology Reporter 12:6-13. https://doi.org/10.1007/BF02668658

Long SP, Zhu XG, Naidu SL, Ort DR (2006). Can improvement in photosynthesis increase crop yields? Plant, Cell \& Environment 29:315-330. https://doi.org/10.1111/j.1365-3040.2005.01493.x

Manivelan K, Karthikeyan M, Blessy V, Priyanka AR, Palaniyappan S, Thangavel P (2019). Studies on correlation and path analysis for yield and yield related traits in greengram [Vigna radiata(L.) Wilczek]. The Pharma Innovation Journal 8:165-167. https://www.thepharmajournal.com/archives/2019/vol8issue9/PartD/8-9-35-920.pdf

Mantel N (1967). The detection of disease clustering and a generalized regression approach. Cancer Research 27:209-220. https://cancerres.aacrjournals.org/content/27/2_Part_1/209

Marappa N (2008). Screening of mungbean genotypes and its wild relatives for resistant sources to Cercospora leaf spot disease. Asian Journal of Biological Sciences 3:324-326.

Mondal MMA, Hakim MA, Juraimi Abdul Shukor, Azad MKA, Karim MR (2011). Contribution of morphophysiological attributes in determining the yield of mungbean. African Journal of Biotechnology 10:1289712904. https://doi.org/10.5897/AJB11.373 
Nagl N, Ajdukovic KT, Barac G, Baburski A, Seccareccia I, Milic D, Katic S (2011). Estimation of genetic diversity in tetraploid alfalfa populations based on RAPD markers for breeding purposes. International Journal Molecular Sciences 12:5449-5460. https://doi.org/10.3390/ijms12085449

Poczai P, Verga I, Laos M, Cseh A, Bell N, Valkonen JPT, Hyvonen J (2013). Advances in plant gene-targeted and functional markers: a review. Plant Methods 9:6-36. https://doi.org/10.1186/1746-4811-9-6

Poolsawat O, Kativat C, Arsakit K, Tantasawat PA (2017). Identification of quantitative trait loci associated with powdery mildew resistance in mungbean using ISSR and ISSR-RGA markers. Molecular Breeding 37:150-161. https://doi.org/10.1007/s11032-017-0753-2

Rhodes I (1972). Yield, leaf area index and photosynthetic rate in some perennial rye grass (Lolium perene L.) selection. The Journal of Agricultural Science 78:509-511. https://doi.org/10.1017/S0021859600026460

Sambrook J, Russel DW (2001). Molecular cloning: a laboratory manual. Cold Spring Harbor Laboratory Press (3rd edn), Cold Spring Harbor, New York, NY, USA.

Shao QS, Guo QS, Deng YM, Guo HP (2010). A comparative analysis of genetic diversity in medicinal Chrysanthemum morifolium based on morphology, ISSR and SRAP markers. Biochemical Systematics and Ecology 38:11601169. https://doi.org/10.1016/j.bse.2010.11.002

Smith JSC, Smith OS (1989). The description and assessment of distances between inbred lines of maize. II. The utility of morphological, biochemical and genetic descriptors and a scheme for testing of distinctiveness between inbred lines. Maydica 34:151-161.

Somta P, Srinives P (2007). Genome research in mungbean (Vigna radiata (L.) Wilczek) and blackgram ( V. mungo (L.) Hepper). Science Asia 33:69-74. https://doi.org/10.2306/scienceasia1513-1874.2007.33 (s1).069

Somta P, Sommanas W, Srinives P (2009). Molecular diversity assessment of AVRDC-the world vegetable center eliteparental mungbeans. Breeding Science 59:149-157. https://doi.org/10.1270/jsbbs.59.149

Srinivasan PS, Chandrababu R, Natarajratnam N, Rangaswamy SRS (1985). Leaf photosynthesis and yield potential in green gram (Vigna radiata (L.) Wilczek) cultivars. Tropical Agriculture (Trinidad) 62:222-224.

Tahir A, Ilyas MK, Sardar MM, Pouya AK, Rasouli F, Bibi A, ... Ghafoor A (2020). Selection criteria for yield potential in a large collection of Vigna radiata(L.) accessions. Euphytica 216:138-149. https://doi.org/10.1007/s10681020-02675-X

Tantasawat P, Trongchuen J, Prajongjai T, Thongpae J, Petkhum C, Seehalak W, Machikowa, T (2010). Variety identification and genetic relationships of mungbean and blackgram in Thailand based on morphological characters and ISSR analysis. African Journal of Biotechnology 9:4452-4464. https://doi.org/10.5897/AJB09.1768

Tantasawat PA, Poolsawat O, Arsakit K, Papan P (2020). Identification of ISSR, ISSR-RGA and SSR markers associated with Cercospora leaf spot resistance gene in mungbean. International Journal of Agriculture and Biology 23:447-453. https://doi.org/10.17957/IJAB/15.1308

Yimram T, Somta P, Srinives P (2009). Genetic variation in cultivated mungbean germplasm and its implication in breeding for high yield. Field Crops Research 112:260-266. https://doi.org/10.1016/j. fc r.2009.03.013

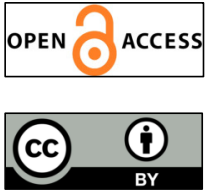

The journal offers free, immediate, and unrestricted access to peer-reviewed research and scholarly work. Users are allowed to read, download, copy, distribute, print, search, or link to the full texts of the articles, or use them for any other lawful purpose, without asking prior permission from the publisher or the author.

License - Articles published in Notulae Botanicae Horti Agrobotanici Cluj-Napoca are Open-Access, distributed under the terms and conditions of the Creative Commons Attribution (CC BY 4.0) License. (c) Articles by the authors; UASVM, Cluj-Napoca, Romania. The journal allows the author(s) to hold the copyright/to retain publishing rights without restriction. 